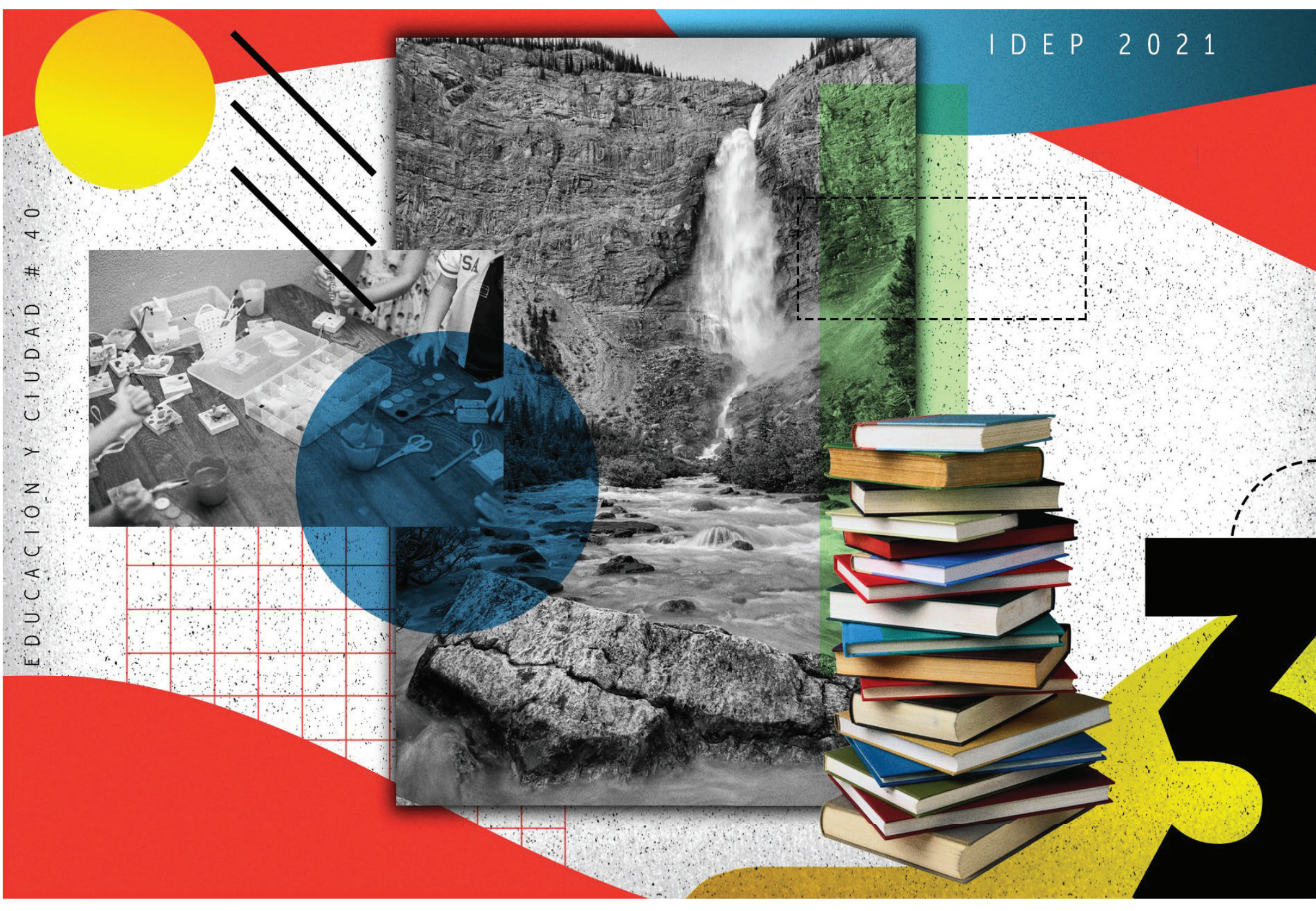

\title{
Agua y procesos de educación ambiental en Bogotá
}

WATER AND ENVIRONMENTAL EDUCATION PROCESSES IN BOGOTÁ ÁGUA E PROCESSOS EDUCAÇÃO AMBIENTAL EM BOGOTÁ

Carmen Alicia Hernández Gómez Claudia María Vargas Aldana 


\section{Carmen Alicia Hernández Gómez ${ }^{1}$}

Claudia María Vargas Aldana ${ }^{2}$
1. Magíster en medio ambiente y desarrollo, Universidad Nacional de Colombia; Docente, Secretaría de Educación de Bogotá; Cvlac: https:// scienti.minciencias.gov.co/cvlac/visualizador/generarCurriculoCv.do?cod_ rh=0001358986; ORCID: https://orcid.org/0000-0001-6266-8063?lang=en; correo electrónico: aliciahgj@gmail.com

2. Candidata a doctora en Ciencias Humanas y Sociales, Universidad Nacional de Colombia; Docente, Secretaría de Educación de Bogotá; Cvlac: http://scienti. colciencias.gov.co:8081/cvlac/visualizador/generarCurriculoCv.do?cod_ rh=0000027981; ORCID: https://orcid.org/0000-0001-7638-757X; correo electrónico: clmvargasal@unal.edu.co

Citar artículo como:

Hernández, C., y Vargas, C. (2021, Enero-Junio). Agua y procesos de educación ambiental en Bogotá. Revista Educación y Ciudad, No. 40, pp. 49-63. //doi.org/10.36737/01230425.n40.2021.2456

DOI: https://doi.org/10.36737/01230425.n40.2021.2456

Fecha de recepción: 3 de agosto de 2020 / Fecha de aprobación: 4 de noviembre de 2020

\section{Resumen}

Este artículo de investigación describe, desde la teoría actor-red, las prácticas de educación ambiental desarrolladas alrededor del agua en distintos escenarios formativos de Bogotá, como los programas de educación de la Secretaría de Ambiente, las instituciones escolares y el trabajo de organizaciones sociales. La descripción onto-epistémica que se propone involucra tres nodos: concepto de agua, estrategias de enseñanza y sentidos pedagógicos; los cuales funcionan para señalar las particularidades que adquiere la enseñanza del agua en espacios formales, informales y no-formales, así, como sus conexiones y colaboraciones.

Palabras clave: Educación ambiental, enseñanza del agua, actor-red.

\section{Abstract}

In this research article, we describe with actor-network theory, the environmental education practices about water in different scenarios in Bogotá: education programs from the Secretary of the Environment, school institutions, and the work of social organizations. The ontoepistemic description that we propose involves three agency nodes: the concept of water, teaching strategies, and pedagogical meanings. These nodes indicate the particularities that the teaching of water acquires in formal and non-formal spaces, as well as its connections and collaborations

Keywords: Environmental education, water education, actor-network.

\section{Resumo}

Neste artigo de pesquisa, é apresentada a teoria ator-rede, para descrever as práticas de educação ambiental na água em diferentes cenários de treinamento em Bogotá: programas de educação do Ministério do Meio Ambiente, instituições escolares e trabalho de organizações sociais. A descrição ontoepistêmica que propomos envolve três nós de agência: o conceito de água, estratégias de ensino e significados pedagógicos. Esses nós indicam as particularidades que o ensino da água adquire em espaços formais e não formais, bem como suas conexões e colaborações.

Palavras-chave: Educação ambiental, educação sobre a água e rede ator. 


\section{Introducción}

$\mathrm{L}_{\mathrm{t}}^{\mathrm{a}}$ a educación ambiental (en adelante EA) permite tejer espacios que llevan a tomar responsabilidades y actuar éticamente en el entorno; en tal sentido, Haraway plantea el reto de participar en la configuración de mundos en los cuales nos sea posible vivir "con", morir "con" y pensar "con" otros de manera recíproca (2019, p. 21); un "con" que no se refiere solo a otras personas, sino a especies y no-humanos. Así, retomar el agua como objeto de enseñanza en tiempos de cambio climático es una manera de tejer compromisos éticos y políticos desde la educación. Un tópico fundamental, porque antes que mera transmisión de conocimientos, los procesos de enseñanza son eventos que implican nuestra participación en la manera de crear una realidad sobre el agua; es decir, la forma de acercarse al tema del agua no solo construye ideas, sino relaciones, acciones y decisiones que se traducen en la capacidad o incapacidad de asumir responsablemente la realidad del agua.

La potencia implícita en la enseñanza del agua desde la EA, y la necesidad de generar reflexiones, retos y provocaciones al respecto, llevó a que, desde la cotidianidad como maestras, se decidiera crear caminos de indagación teórica y práctica en torno a la forma como el concepto agua fluye en las aulas y la institucionalidad de la escuela; esperando que este ejercicio de indagación, construido desde el ser y estar en el mundo como maestras, aporte, no solo al propio quehacer, sino al de otros maestros con quienes caminamos sin conocernos. Justamente, uno de esos caminos de indagación se presenta en este artículo, buscando dar cuenta de las maneras como el agua, en tanto objeto de enseñanza, se construye en las prácticas de EA formal y no formal en la ciudad de Bogotá.

Puesto que la pregunta gira alrededor de la construcción de un objeto de enseñanza no neutral, y responde a un escenario muy complejo, se retoma la teoría del actor red, trabajada por Callon (1998), Latour (2008) y Law (2019), que invita a describir prácticas y relaciones desde la apertura, el carácter situado y la especificidad de las realidades abordadas. A continuación, se exponen algunas líneas de referencia propuestas por dichos autores, que contribuyen a situar el marco de esta investigación:

- El sentido de la investigación se concentra en las redes constituidas por relaciones, prácticas y distintas entidades que intervienen.

- Las redes no son estáticas, se encuentran en medio de un proceso de cambio, en el cual son hechas y crean los objetos, prácticas y relaciones, que también se encuentran en un círculo de estar y desaparecer.

- La agencia social no es exclusiva de humanos, involucra también objetos, entidades y seres no-humanos.

- Se presenta un principio de simetría en el abordaje de la producción de conocimiento.

A partir de las líneas de referencia, y de los materiales empíricos que sustentan esta investigación, se proponen tres nodos que articulan de distintas maneras las prácticas educativas sobre el agua en Bogotá: conceptos sobre agua, estrategias pedagógicas y sentidos pedagógicos.

Los conceptos de agua se refieren a los saberes, conocimientos, discusiones y debates provenientes 
de disciplinas, ciencias y tradiciones que permiten relacionarse con el agua, ya sea desde su acepción compuesto químico, como recurso o como entidad espiritual y sagrada. Las estrategias pedagógicas son un conjunto de herramientas que posibilitan la interacción o incorporación de esos conceptos en las prácticas; y los sentidos pedagógicos recuerdan que la enseñanza y el aprendizaje están atravesados por relaciones de poder que implican el gobierno de las poblaciones (Noguera y Parra, 2015, p. 72); así, referirse a los sentidos significa enfocarse en el ciudadano que deviene en la práctica educativa. Los tres nodos operan como objetos aprendidos e incorporados, con ellos es posible enseñar de manera específica el tema del agua; además, permiten resaltar que la práctica pedagógica no solo posee un carácter epistemológico, sino material y, por esta vía, ontológico.

Con ello en mente, el artículo se divide en cuatro secciones: en primer lugar, la revisión de la literatura académica que aborda cómo circula el agua en la educación y las discusiones alrededor de su enseñanza; en segundo lugar, se da cuenta de las precisiones metodológicas que guían la investigación y permiten retomar los nodos mencionados anteriormente; en tercer lugar, se encuentra el apartado de análisis, el cual retoma los conceptos, estrategias y sentidos pedagógicos que se articulan y emergen en distintas prácticas pedagógicas de Bogotá, desa-

3 Entre los principios de la EA es posible encontrar: un enfoque sistémico; valor de diversidad biológica y cultural; nuevo concepto de necesidad; desarrollo de conciencia local y planetaria; solidaridad; estrategias democráticas; valor educativo de conflicto; valores como fundamento de la acción; pensamiento crítico e innovador; integración de conceptos, actitudes y valores (Novo, 1996, p. 91). rrolladas por entidades distritales, organizaciones sociales e instituciones educativas distritales; finalmente, se realiza un cierre, destacando los vínculos generados desde las tres prácticas en la composición compleja de los tejidos que articulan la circulación del agua en las escuelas de Bogotá.

\section{Agua y educación: una revisión de la literatura académica}

Hasta el momento, los trabajos consolidados alrededor del agua y la educación ambiental se abordan partiendo de cinco ejes: el campo de lo ambiental como conocimiento vs los campos "científicos" del saber tradicional; el qué y cómo enseñar del agua; su relación con el concepto de naturaleza; el discurso científico sobre el agua presente en los textos escolares; y las formas como el agua se constituye en un elemento de interrelación del paisaje y la construcción de territorio.

La literatura también da cuenta de una variedad de énfasis, debates y desplazamientos que indican cómo la educación ambiental, en tanto campo de saber escolar, se encuentra en medio de la tensión entre la apertura y lo instituido. Aunque existen una serie de principios que caracterizan la $\mathrm{EA}^{3}$, este campo de saber escolar no solo presenta una reciente emergencia, sino que todavía está en pro- 
ceso de formalización, pues sus metodologías, contenidos y sustentos teóricos y científicos proceden de una serie de campos científicos fronterizos e interdisciplinares. Así, la EA se sitúa en un momento de búsquedas, préstamos y experimentaciones que pueden llevar, tanto a la innovación pedagógica, como a la acomodación en zonas de confort disciplinares.

Por ello, si se pretende constituir una EA innovadora, crítica y pertinente, es necesario prestar atención a los trabajos que abordan las concepciones, representaciones e imaginarios tejidos alrededor del agua en las comunidades educativas, en los sujetos de la enseñanza y el aprendizaje, y en los libros de texto, destacando especialmente tres puntos, siendo el primero de ellos el debate sobre cuáles deben ser los ejes temáticos y problemas que articulan la enseñanza del agua. Lo cual implica un llamado para que, en general, los procesos de educación ambiental sobre el agua dejen de centrarse en la calidad del líquido, para empezar a abordar los ecosistemas de agua y las situaciones que les constituyen (Ibarra, 2007); de tal forma que las referencias no apunten solo a sus características químicas, su presencia o ausencia, o al ciclo hidrológico, sino que incluyan situaciones problemáticas y unidades didácticas contextualizadas (Marcen, 2003).

Siguiendo esta línea, durante los últimos años ha sido posible observar que los estudios sobre EA empiezan a retomar las experiencias situadas en problemáticas y conflictos ambientales que giran en torno a distintas fuentes hídricas. Tales trabajos plantean la necesidad de romper con una enseñanza basada en el inventario de características y datos sobre el agua y fuentes hídricas, para empezar a fortalecer una mirada contextual y crítica entre los sujetos de enseñanza y aprendizaje, el conocimiento y el territorio que habitan.

Dichas nuevas formas de relacionarse con el agua se encuentran en ejercicios pedagógicos sistematizados que, para el caso de Bogotá, trabajan la cuenca del Río Tunjuelo, como en Hernández y Vargas (2015), quienes centran su discusión en el río, la minería como conflicto y la formación política con jóvenes de la localidad de Ciudad Bolívar; junto a ellos, Castaño y Urrego (2015) abordan, desde la enseñanza para la comprensión, la identificación de los distintos conflictos ambientales en la cuenca del Tunjuelo y, Barrera (2019) propone, no solo su identificación, sino la consolidación de redes de participación en una institución escolar, para contribuir con el cuidado del río.

El segundo eje del debate se relaciona con la manera de entender la relación entre hombre y naturaleza, pues las representaciones y concepciones de maestros, estudiantes y comunidades tienden a hacer de ella un recurso, a pesar de que se han establecido otras relaciones simbólicas para este vínculo, por lo general invisibilizadas y/o subvaloradas; para el caso, conviene recordar lo planteado por Flórez (2009), quien, al estudiar las representaciones sobre el agua y su cuidado en maestros en formación, destaca que la mayoría de ellos presenta una mirada utilitarista; en la misma línea, Ramírez (2016), resalta que, en el caso de estudiantes rurales, aunque el agua es identificada como sinónimo de vida, suele entendérsele como elemento distante, reforzando una mirada dualista de dicha relación.

El tercer debate gira alrededor de la forma de trabajar el agua en los libros de texto; las investigaciones que retoman el escenario presentan diversas 
críticas a la manera de presentar el conocimiento científico, pues destacan aspectos problemáticos en relación con su transposición o simplificación, los cuales deben tenerse en cuenta para fortalecer los materiales puestos a disposición de los estudiantes; como ejemplo, Moss y Mizuno (2011), partiendo del análisis semiótico discursivo de un libro escolar en Colombia, destacan que, cuando se refieren al agua, las voces de los autores presentan el conocimiento como verdad absoluta, sin ofrecer argumentos, debates o citas, por lo que no hay una contextualización sobre la producción de saber, o de quiénes y por qué lo producen, lo cual tiene efectos en la manera en que los estudiantes se acercan y entienden la generación de conocimiento científico sobre el agua.

En tanto, un estudio español sostiene que, durante el abordaje de las fuentes hídricas como ríos, los libros de texto mantienen miradas tradicionales, reduciéndolas a meros recursos mercantiles, cuando incluso los conceptos y conocimientos hidrológicos manejados ya han sido reevaluados por las ciencias (Cuello y García, 2019). Mientras un análisis histórico realizado por Moctesuma plantea que, entre fines del siglo XIX e inicios del siglo XX, los libros de texto mexicanos, desde su lugar como objeto cultural, abordan el agua a partir de los discursos de progreso y modernización promovidos por el Estado, constituyéndose en herramientas para instituir procesos de higienización y "obrerización" en la población, fortaleciendo la identidad nacional en torno a la riqueza hídrica y el desarrollo de procesos de gestión sobre el agua, como el secamiento de sus fuentes, lo cual se presentaba como hecho necesario para el "progreso" (Moctezuma, 2006).
Finalmente, aparecen los estudios que retoman procesos de sistematización de experiencias relacionadas con el cuidado, calidad y gestión del agua, junto con las diversas interacciones biológicas, sociales, históricas y culturales inherentes a ella y las fuentes hídricas, posicionando formas participativas de abordar el tema en la EA. Esta corriente genera dos tendencias de inclusión de las comunidades en la educación ambiental escolar y no formal; por un lado, se crean procesos centrados en objetos de conocimiento producidos por la ciencia participativa, como las experiencias en que los participantes aprenden a medir las características físicas y químicas del agua como forma de apropiación del territorio, y de seguimiento al estado de las fuentes hídricas que alimentan los acueductos o están cerca de las instituciones escolares (Hurtado, 2019).

Por otro lado, se encuentran procesos en torno a la construcción de objetos de conocimiento desde los saberes y necesidades de las comunidades; así, ellas participan en procesos de EA, ya sea para potenciar a partir de sus saberes los procesos de gestión del agua (Silva, Alatorre y García, 2009), para fortalecer las competencias en el cuidado de las fuentes hídricas (Moreira, Araya y Charpentier, 2015; Lara y Otaño, 2017), o para ser referentes de conocimientos sobre la memoria y cuidado del agua a partir de procesos de comunicación intergeneracionales e interculturales (Rubio, 2017; Herrera, 2017; Lemus, 2018).

\section{Metodología}

Esta indagación es de tipo cualitativo y retoma un análisis praxiológico para acercarse a las prácticas de educación ambiental sobre el agua en Bogotá. La praxiología se centra en "prestar atención obs- 
tinadamente a las técnicas que hacen que las cosas sean visibles, audibles, tangibles y conocibles" (Mol, 2002, p. 33), por lo que permite enfocarse en las distintas maneras en que maestros, estudiantes, funcionarios de entidades públicas, líderes sociales y no-humanos se articulan a través de los nodos "agenciales" (presentados en la sección anterior), para materializar unas formas específicas de enseñar sobre el agua en Bogotá.

En esta vía, la praxiología exige que la construcción de materiales empíricos se realice atendiendo a las maneras en que los distintos agentes concretan sus prácticas, no a las formas en que las representan. Así, desde el año 2019, hasta el mes de julio de 2020, se retomaron, como herramientas de investigación, la realización de entrevistas a docentes del distrito, la observación en escenarios educativos escolares y, en el marco de la emergencia sanitaria decretada para contener el Covid-19, herramientas de la etnografía virtual que, en este caso, giraron alrededor de la participación en los distintos espacios públicos de educación virtual ofrecidos por la Secretaría de Ambiente en Facebook Live y video llamadas.

\section{Análisis o resultados}

A partir de los nodos "agenciales", el presente apartado girará alrededor de los siguientes tópicos: concepto de agua; estrategias pedagógicas y sentidos pedagógicos; revisando la forma como tres escenarios de educación ambiental establecen distintas relaciones que configuran, en cada uno, de manera particular, no aislada, una serie de vínculos concretos, los cuales caracterizan la red que constituye el agua como objeto de enseñanza de la educación ambiental; dichos espacios son a) Algunos proce- sos de educación informal liderados por organizaciones sociales; b) La educación no formal, como el programa virtual de educación ambiental de la Secretaría de Ambiente; y c) La educación formal impartida desde la escuela.

\section{Organizaciones sociales, afectos y cuerpos de agua}

El agua es un elemento que implica contradicciones, conflictos y respuestas sociales donde los actores (re)configuran cuadros culturales de referencia y relaciones político-económicas con símbolos o signos. El concepto de agua se teje desde las relaciones instituidas y construidas por la cultura, y su carácter físico se traslapa en las diferentes territorialidades que constituye; así, es centro de procesos de gobernanza territorial, resistencias y per-existencias.

Distintas organizaciones sociales han asumido el cuidado de cuerpos de agua como ríos y humedales a partir de la educación ambiental; reconocerlos como parte de sus espacios vitales configuró nuevas formas de crear tejido social en torno a ellos, transformando el lente a través del cual se valoraban. Iniciativas como las implementadas por la Fundación Crisálida y Territorio Sur en el Tunjuelo, o los Amigos del río, en el Fucha, tienen apuestas en común respecto a su accionar organizativo; habitantes del territorio asumen posturas críticas y participativas frente a las transformaciones o intervenciones de los cuerpos de agua, consolidándose como grupos intergeneracionales con los cuales se elaboran narrativas propias del concepto agua.

Sus estrategias pedagógicas giran en torno a recorridos de las cuencas, la identificación de fauna y 
flora presente, actividades de reforestación, círculos de palabra ancestral y actividades artísticas de reconocimiento de los sentidos espirituales del agua. Dichas tácticas configuran procesos de educación ambiental, cuyos sentidos pedagógicos son buscar la sensibilización y conciencia de los habitantes del sector para permitirles "ser parte de" y así generar ejercicios políticos de participación y corresponsabilidad en las decisiones que se tomen frente a los cuerpos de agua.

En la misma línea, otra organización destacable es la Fundación de Humedales de Bogotá, que desde 1998 realiza un trabajo pedagógico en torno a la preservación de este ecosistema; sus actividades retoman el valor del agua como fuente de biodiversidad y, además de dar cuenta de qué es un humedal y sus características, sus talleres al aire libre resaltan la relación entre el agua y las especies vegetales, animales y de hongos. El sentido pedagógico no solo trabaja la preservación del ecosistema, busca generar una relación profunda entre comunidades y humedales; para ello, retoma estrategias que incluyen estar en el lugar y, desde la práctica, conocer y generar afecto, pues: "el amor hacia el ecosistema, una planta, un animal o un hongo hace que trabajemos para conservarlo" (Entrevista a integrante de la Fundación de Humedales).

Prácticas educativas como las construidas por estas organizaciones sociales permiten generar relaciones y vínculos emocionales con aquello no humano, como estrategia de apropiación de los territorios y de preservación de los ecosistemas, conectándose así con los procesos de educación ambiental formal, no solo porque cuentan con participación de estudiantes y maestros, sino por sus efectos en la organización curricular; de acuerdo a varias entre- vistas con maestros, su interés por abordar desde la clase los cuerpos de agua cercanos al colegio, está profundamente relacionado con su participación en procesos de educación ambiental informal o, como afirma Flórez (2009), de educación ambiental popular.

\section{Apuestas pedagógicas desde la Secretaría Distrital de Ambiente (SDA)}

Por su parte, frente a las instituciones gubernamentales distritales, cuya misión permite realizar acciones sistemáticas de EA, ha sido posible encontrar que el trabajo virtual es el nodo que articula los procesos educativos, pues permite, de acuerdo con algunos de sus promotores, un mayor acceso a distintas poblaciones. Así, aunque la llamada "cuarta revolución" ha transformado todas las actividades del ser humano, en la estructura de la educación colombiana se venían articulando de modo paulatino diferentes tecnologías; sin embargo, las circunstancias presentadas durante el 2020, a causa de las medidas adoptadas para neutralizar la propagación del COVID-19, han generado un aceleramiento desafiante en la implementación general de las TIC en la educación.

En tal contexto, la EA se instala como disciplina híbrida desde la perspectiva pedagógica, epistemológica y ética; se propone re-orientar el curso en favor de una comprensión diferente y compleja de la relación sociedad-naturaleza. Este proceso de actualización educativa supone, entre otros instrumentos, la introducción de las TIC mediante la capacitación docente y la masificación de programas de EA al público general. Debido a que ella tiene un rol fuertemente ético, las TIC no deben ser integradas al proceso educativo irreflexivamente, deben 
ayudar a romper la lógica generada por los problemas ambientales contemporáneos, es decir, se trata de una herramienta que ayuda al desarrollo social desde la solución de los problemas de la EA (Corbeta, Sessano y Krasmanski, 2012).

Las clases virtuales de educación ambiental de la SDA fueron creadas el 6 de abril, con el objetivo de involucrar a los ciudadanos en los procesos de educación ambiental que buscan la apropiación social del territorio, de los escenarios ambientales y la defensa de la vida en todas sus formas, de acuerdo con los principios de la Política Pública de Educación Ambiental (SDA, 2007). Los eventos se desarrollan de forma semanal a través de diferentes plataformas, donde los temas principales son biodiversidad, cambio climático, gestión integral de residuos, gestión de riesgos, programas posconsumo y territorios ambientales.

Dentro de las clases virtuales se desarrolla una serie de sesiones alrededor del agua en Bogotá. La red se teje desde la idea de que se trata de un bien público de la ciudad, por lo que implica una serie de conexiones temáticas como: el ciclo hidrológico y el papel de los páramos en su producción; la estructura ecológica principal de Bogotá; el sistema de abastecimiento de agua potable; el sistema de alcantarillado y los vínculos ancestrales entre el líquido y las culturas indígenas y campesinas de la ciudad.

A partir del desarrollo de estas temáticas mediante cátedras magistrales por video llamada, presentación de videos que destacan los distintos cuerpos de agua, y reflexiones de profesionales a cargo, se busca generar en los asistentes el respeto por el agua e incentivar su preservación. Así, esta red de educación ambiental se articula con la formación de ciudadanos que, mediante el cuidado y preservación del agua, contribuya a sostener en el tiempo los servicios ecosistémicos y culturales brindados por ella a la ciudad.

Los programas y estrategias pedagógicas de la SDA profundizan en un concepto del agua que transita, desde el concebirla como bien público, hasta verla como bien simbólico ancestral, complejizando los saberes que la vinculan con la vida. Ello determina sentidos pedagógicos más profundos, que movilizan, desde otras maneras de "hacer" agua, las responsabilidades del individuo frente al ambiente; por ejemplo, entre las propuestas de EA promovidas por la SDA se destaca AUAMBARI, aula ambiental artística itinerante que busca llevar EA a todos los bogotanos mediante el arte y el conocimiento ancestral.

Willington Rocha, líder del aula, y diez representantes de grupos étnicos, con diferente formación profesional, conforman un equipo pedagógico que da vida al aula, unidos por el mismo sentido pedagógico: transitar la zona urbana y rural llevando un mensaje de sostenibilidad a través del arte, incorporando las visiones ancestrales, étnicas y el conocimiento ambiental (SDA, 2020). El aula existe desde 2013, pero la pandemia llevó a realizar un cambio hacia el mundo virtual, enfocándose en la categoría de la música; aseguran que dicha transformación fue compleja porque no todos tienen acceso a un computador, pero, al tiempo, destacan que han llegado a más personas por medio de las redes sociales, especialmente a través de la página de $\mathrm{Fa}-$ cebook “@AmbienteBogota”. 


\section{Instituciones escolares: entre estándares y el fluir del agua en el aula}

La escuela suele entenderse como una organización cerrada y estática, no obstante, ofrece espacios de apertura en el marco de los diálogos que entabla con sus contextos políticos, económicos y sociales; de ahí que las prácticas de EA, desarrolladas en ella, proponen una tensión, entre las lógicas que reproducen el dualismo hombre/naturaleza, y aquellas que lo cuestionan. La lógica del dualismo se expresa en hechos como que la estructura y el orden disciplinario escolar se fundan desde la fragmentación y el mecanicismo de la ciencia moderna, cuyo imaginario implica una relación simbólica con la naturaleza y lo no humano en términos de supremacía, mercantilización y desidia, sobrevalorando la "tecnociencia", el progreso y el valor económico.

Las entrevistas con los maestros indican que dicha lógica se hace evidente en el lenguaje de los estándares básicos de la educación, pues en ellos prevalecen los términos de uso, desarrollo, progreso, recurso y apropiación del agua. Además, no contemplan las dinámicas sociales implícitas en lo ambiental, el entramado de relaciones de diferentes actores con la naturaleza, o lo ambiental entendido como las interrelaciones hombre-naturaleza mediadas por la cultura. Los estándares hacen invisible la multiplicidad de actores y sinergias presentes en un país diverso, priorizando los modelos hegemónicos y eliminando, para ello, la complejidad y la problematización.

Por su parte, la segunda lógica entiende que la escuela es un escenario que da lugar a muchos espacios, constreñidos por los problemas cotidianos, las carencias y ausencias, pero, al tiempo, se trata de un contexto diverso y creativo, desde la multiplicidad de voces e historias que le habitan, pero ¿Cómo se instala la palabra agua en esa cotidianidad?; ¿cómo se incorpora nuestra identidad de país húmedo, anfibio y fluido en la formación del ciudadano?; ¿cuáles lenguajes determinan los discursos sobre el agua en la escuela?

Las respuestas pueden ser infinitas. No obstante, a partir de los materiales empíricos construidos, fue posible encontrar dos formas de articulación de redes, que gravitan alrededor del nodo del sentido pedagógico. Es decir, las prácticas escolares analizadas se conectan a partir de dos maneras de formar ciudadanos, la cuales no se excluyen necesariamente. La primera articulación surge alrededor de la formación en actitudes personales que tiendan al cuidado del agua; para ello, el agua se aborda desde sus procesos físicos y químicos, reconociendo la contaminación como problemática ambiental, la huella hídrica y su relación con la vida y los usos humanos. Aquí, la principal estrategia es la conmemoración del Día del Agua, que permite plantear distintos procesos reflexivos y actividades artísticas, en las que la escasez y la contaminación son los temas recurrentes.

Junto a esa actividad, otras estrategias retomadas en las instituciones escolares son la creación y disposición de carteles alusivos al cuidado del agua en distintos espacios físicos del colegio; en algunas clases se realizan ejercicios que parten del análisis de los recibos del servicio de agua, para concienciar respecto del uso personal del líquido. En las dos estrategias el cuidado se traduce en procesos de racionalización y ahorro que los estudiantes deben incorporar a su cotidianidad; al tiempo que ideas 
como el valor de las fuentes hídricas, el agua es vida, o la referencia a que "somos agua".

Por su parte, en la segunda articulación las prácticas apuntan a generar compromisos comunitarios y procesos de participación para el cuidado y defensa del agua, los territorios y ecosistemas, por lo que se opta por prácticas pedagógicas al aire libre, donde se da la posibilidad de interactuar con ecosistemas no humanos, miembros de organizaciones sociales y la comunidad en general. Una labor que implica ejercicios académicos constantes en el tiempo, durante los cuales se resaltan aspectos como los vínculos biológicos, culturales, sociales, económicos y emocionales presentes en las relaciones entre agua y comunidades. De acuerdo con las entrevistas y observaciones realizadas, estas prácticas no suelen estar vinculadas al PRAE y surgen de procesos interdisciplinares entre las áreas de ciencias naturales, ciencias sociales y, en algunas ocasiones, humanidades y artes; así:

La escuela está siendo repensada y transformada en una trinchera de la Educación Ambiental como práctica social y política, integrando la participación comunitaria como apuesta fundamental dentro de la sensibilización y apropiación con relación a las problemáticas de índole ambiental (García y Ruíz, 2008).

\section{Conclusiones}

Una EA crítica, contextualizada y construida con distintos sectores, no solo funciona como herramienta para rescatar a la naturaleza de la hecatombe en términos físicos, sino para salvar al ser humano del abismo al que le han conducido las relaciones históricas. Liberar al planeta significa luchar por los manglares, los ríos, el cielo, los océanos, etc., pero también pelear por el ser humano en sí mismo; si se quiere separarse de la división moderna de humano-naturaleza, es necesario reconocer que en esta crisis el ser humano también debe ser salvado, que de nada servirá rescatar a la naturaleza si no se le libera en medio y a través del proceso.

Las prácticas de educación ambiental sobre el agua presentan un desplazamiento importante, pues transitan, desde una enseñanza centrada en los paradigmas científicos que reproducen el dualismo hombre/naturaleza, hasta una enseñanza más compleja que logra articular distintos saberes e incorporar relaciones de saber y afectivas con actores no humanos, como ríos y humedales. Este fenómeno, cuya consolidación parte de principios de 2000, reporta interesantes avances en el cambio de las formas de abordar lo ambiental, específicamente el agua, en la escuela. Existen múltiples experiencias en colegios distritales donde las prácticas pedagógicas dan cuenta de la complejidad de lo ambiental, a partir de discursos que ponen en diálogo diferentes saberes, ontologías y gnoseologías, en el marco de una escuela abierta donde diferentes actores humanos, y no humanos, comienzan a tejer su identidad y territorio.

Este desplazamiento es fuerte en las instituciones escolares gracias a los diálogos y vínculos entre escuela y procesos de educación ambiental informal y no formal. Así, en Bogotá, se encontró que se tejen procesos de articulación alrededor de la enseñanza del agua, en los cuales la apropiación del territorio, el cuidado de cuerpos de agua y los saberes ancestrales y campesinos potencian procesos de formación de ciudadanías, incorporando el agua en la ciudad de manera compleja y relacional. 


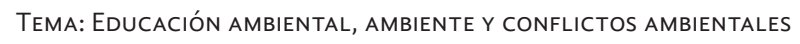

Así mismo, a pesar de que se mueven en distintos escenarios, estas redes presentan comunicaciones importantes, por lo que es necesario un trabajo interinstitucional que reflexione, proponga y lidere un trabajo conjunto, de tal manera que perduren en el tiempo y no se enfoquen en actividades dispersas e inconexas. 道 


\section{Referencias}

Barrera, E. (2019). Aportes de la participación ciudadana de niños y niñas de octavo grado del CED Jackeline en el cuidado ambiental de la cuenca del Rio Tunjuelo. Tesis de Maestría en educación no publicada, Pontificia Universidad Javeriana, Bogotá.

Callon, M. (1998). El proceso de construcción de la sociedad. El estudio de la tecnología como herramienta para el análisis sociológico. En Doménech, M., y Tirado, F. J. Sociología Simétrica. Ensayos sobre ciencia, tecnología y sociedad (pp. 143 - 170). Barcelona: Gedisa.

Castaño, M., y Urrego, L. (2015). Problemáticas socioambientales en la cuenca del Río Tunjuelo: una posibilidad en la enseñanza y el aprendizaje de la geografía. Giramundo, 2(4), pp. 95-108.

Corbetta, S., Sessano, P., y Krasmanski, M. (2012). Educación Ambiental (EA), formación docente y TIC's, el desafío complejo de una triple articulación. Hacia la definición de un espacio transversal. Revista de la escuela de ciencias de la educación, No. 7, pp. 267-290.

Cuello, A., y García, F. (2019). ¿Ayudan los libros de texto a comprender la realidad fluvial de la ciudad? Revista Humanidades, No. 37, pp. 209-234. DOI: https://doi.org/10.5944/rdh.37.2019.22895

Flórez, R. (2009). Las representaciones sociales del uso del agua. Memorias electrónicas del X Congreso Nacional de Investigación Educativa. México: Consejo mexicano de investigación educativa.
García, M., y Ruíz, D. (2008). El debate discursivo modernidad-posmodernidad y la educación ambiental en la escuela contemporánea. Educere, 12(42), pp. 487-494.

Haraway, D. (2019). Seguir con el problema. Generar parentesco en el Chthuluceno. Bilbao: Edición Consonni.

Hernández, A., y Vargas, C. (2015). Minería y cambio climático en la cuenca del Tunjuelo: una experiencia de formación política en la escuela. Revista Educación y Ciudad, No. 30, pp. 99-106.

Herrera, Y. (2017). La transformación del discurso acerca de las problemáticas del Río Bogotá de los estudiantes del grado $7 b$ de la I.E. Santa María del Río. Tesis no publicada, Universidad Pedagógica Nacional, Bogotá.

Hurtado, R. (2019). La educación Remona. En Palacio, D., Van der Hammen, M., y De Urbina, A. (Eds.), Fuentes vivas en el borde [e-book]: investigación y experiencias colaborativas para la gobernanza de un sur sostenible en Bogotá (pp. 3-19). Bogotá: Universidad Externado de Colombia. Centro de Educación Virtual. DOI: https://doi. org/10.18800/educacion.201901.005

Ibarra, J. (2007). Nuevos contenidos educativos sobre el agua y los ríos desde una perspectiva CTS. Revista Electrónica de Enseñanza de las Ciencias, No. 6, pp. 714-728. 
Lara, A., y Otaño, C. (2017). Agua, educación y cultura. Trabajo presentado en el XXVI Congreso Nacional del Agua, Argentina.

Latour, B. (2008). Reensamblar lo social: una introducción a la teoría actor-red. Buenos Aires: Manantial.

Law, J. (2019-Marzo 15). Actor Network Theory and Material Semiotics. Obtenido desde http:// www. heterogeneities. net/publications/ Law2007ANTandMaterialSemiotics.pdf

Lemus, J. (2018). El cuidado del agua, Una propuesta pedagógica de educación ambiental, desde la perspectiva biocéntrica, basada en la cosmovisión de las etnias Cubeos, Jiw, Piratapuyos y Tuyucas. Tesis de Doctorado no publicada, Universidad Santo Tomás, Bogotá.

Marcen, C. (2003). Aportaciones desde la escuela a la nueva cultura del agua. Agua y educación ambiental: nuevas propuestas para la acción (pp. 2530). Madrid: Caja de ahorros del Mediterráneo.

Ministerio de Educación Nacional (MEN). (2004). Formar en ciencias: ¡El desafío! Obtenido desde http://eduteka.icesi.edu.co/pdfdir/MENEstandares CienciasSociales2004.pdf

Moctesuma, L. (2006). El agua y los recursos naturales como representación en libros de texto mexicanos, 1882-1920. Revista mexicana de investigación educativa, 11(30), pp. 847-866.
Mol, A. (2002). The body multiple: ontology in medical practice. Durham: Duke University Press. DOI: https://doi.org/10.1215/9780822384151

Moreira, C., Araya, F., y Charpentier, C. (2015). Educación ambiental para la conservación del recurso hídrico a partir del análisis estadístico de sus variables. Revista Tecnología en Marcha, 28(3), pp. 74-85. DOI: https://doi.org/10.18845/ tm.v28i3.2413

Moss, G., y Mizuno, J. (2011). Las voces del texto. En Barletta, N., y Chamorro, D. (Eds.), El texto escolar y su aprendizaje, enredos y desenredos (pp.149-179). Barranquilla: Universidad del Norte.

Noguera, C., y Parra, G. (2015). Pedagogización de la sociedad y crisis de la educación. Elementos para una crítica de la(s) crítica(s). Pedagogía y Saberes, No. 43, pp. 69-78.

Novo, M. (1996). La educación ambiental formal y no formal: dos sistemas complementarios. Revista iberoamericana de educación, No. 11, pp. 75-102.

Ochoa, A., García, A., Carvajal, M., y Ríos, C. (2017). Percepciones de la comunidad educativa del Porvenir, Rionegro, Antioquia, sobre la importancia de los servicios ecosistémicos del Río Negro. Bio-grafía. Escritos sobre la Biología y su Enseñanza. Memorias del IX Encuentro Nacional de Experiencias en Enseñanza de la Biología y la Educación Ambiental, pp. 121-133. DOI: https:// doi.org/10.17227/bio-grafia.extra2017-7090 
Ramírez, V. (2016). Representaciones sociales del agua y medio ambiente en los niños, un análisis desde la educación ambiental en un contexto rural. Tesis de grado no publicada, Pontificia Universidad Javeriana, Bogotá.

Rubio, P. (2017). Los habitantes del río: construyendo caminos para la enseñanza-aprendizaje del cuidado de la vida, una experiencia de práctica pedagógica integral enmarcada por el ecosistema de río y la cultura anfibia en la institución educativa San Francisco de Loretoyacu y comunidades ubicadas a lo largo de la desembocadura del Río Loretoyacu, Boyahuazú y Atacuari. Tesis de grado no publicada, Universidad Pedagógica Nacional, Bogotá.

Secretaría Distrital de Ambiente (SDA). (2007). Política Pública Distrital de Educación Ambiental. Obtenido desde http://ambientebogota.gov.co/c/ document_library/get_file?uuid=63781256-a5ed4136-ae04-e6708fdb7f0e\&groupId=55886

Secretaría Distrital de Ambiente (SDA). (2020-Junio). Aula ambiental artística AUAMBARI. Obtenido desde http://www.ambientebogota.gov. co/web/sda/historial-de-noticias/-/asset_publish er/1RkX/content/canciones-para-aprender-a-cui dar-el-ambiente\%3A-un-aula-ambiental-disponibl e-para-todos? redirect=http $\% 3 \mathrm{~A} \% 2 \mathrm{~F} \% 2 \mathrm{Fwww}$. ambientebogota.gov.co\%2Fweb\%2Fsda\%2Fhistori al-d

Silva, E., Alatorre, G., y García, H. (2009). Formación ambiental e investigación: un enfoque metodológico plural para la gestión de las cuencas, los bosques $y$ el agua en Veracruz. Trabajo presentado en el X
Congreso Nacional de Investigación Educativa, México, Consejo mexicano de investigación educativa. 